\title{
Effect of Peptones and Study of Feeding Strategies in a CHO Based Fed-batch Process for the Production of a Human Monoclonal Antibody
}

\author{
Véronique Chotteau, Caroline Wåhlgren, Helena Pettersson \\ Biovitrum, Biopharmaceuticals, Process Development, Process Sciences, Stockholm, Sweden
}

\begin{abstract}
Eight commercial peptones, derived from plants, were studied for their ability of improving the cell growth and the productivity of a $\mathrm{CHO}$ cell line producing a human monoclonal antibody. They were also compared to yeast, lactalbumin and meat derived peptones. Seven plant peptones were selected and further studied in combination by Design of Experiment. The best three peptones were then tested in combinations in fed-batch cultivation. The fed-batch process was based on low concentrations of glucose and glutamine with feeding of amino acids, peptones and feed medium including vitamins, metal traces and biosynthesis precursors. This process was based on Biovitrum protein-free proprietary medium for the base medium and the feeding medium. Different feeding strategies, different peptone combinations and phosphate feeding were studied for their ability to improve the cell density, the cell specific productivity and the cultivation longevity.
\end{abstract}

Key words: peptone, protein hydrolysate, $\mathrm{CHO}$, Chinese Hamster Ovary, fed-batch, feed, DoE, Design of Experiment, antibody, MAb, soy, wheat, rice, pea, yeast, cotton, Primatone, lactalbulmin, phosphate 


\section{RESULTS AND DISCUSSION}

\subsection{Screening of various peptones, dosage study and combination study}

The effect on the cell growth and the productivity of 8 plants peptones (HyPep 1510 soy, HyPep 4601 wheat and HyPep 4605 wheat, HyPep 5603 rice, HyPep 7401 pea, HyPep 7504 cotton seed, all Kerry, and P-0521 soy Sigma, Soy Protein Acid Hydrolysate Sigma) and 1 yeast peptone (HyPep 7455 Kerry) was studied including a comparison with Primatone meat Kerry and HyQ lactalbumin cow milk Hyclone. The results indicated that 5 plant peptones resulted in the best cell growth and productivity at levels comparable to the ones obtained by using Primatone and that the best results were obtained between 5 and $10 \mathrm{~g} / \mathrm{L}$ peptone with worse results at $1 \mathrm{~g} / \mathrm{L}$ and $11 \mathrm{~g} / \mathrm{L}$ peptone and above. Combinations of 2 or 3 peptones of the 5 selected peptones plus 2 additional peptones were studied by Design of Experiment; the total peptone concentration being $5 \mathrm{~g} / \mathrm{L}$. From this, 3 plant peptones, coded PEP D, E and G, were selected for their effect on the cell growth, the viability or the productivity. No synergy effect or detrimental effect of combining the peptones were observed in this experiment.

\subsection{Fed-batch cultivation with peptone addition}

Diverse combination of peptones PEP D, E and G in the base medium and the feed were studied in fed-batch spinners as well as feeding of phosphate. Comparing feeding or not the feed medium while glucose, glutamine and amino acids were fed showed that the feed medium addition was beneficial. It was showed that the presence of peptone PEP G resulted in better productivity and growth and that feeding phosphate did not improve the process. An early unexplained decline in viability was observed reproducibly in two run series despite the fact that the by-product accumulation or the osmolarity were not too high. A screening was then performed in 6-wells plate system showing that the early viability decline was due to the simultaneous feeding of feed medium, peptones D and $\mathrm{E}$ and amino acids. The feed medium composition was also slightly modified and a new series of fed-batch runs in $100 \mathrm{ml}$ spinners was performed to compare the effect of different combinations of 2 peptones from PEP D, E and G in the base medium (BM), the effect of feeding frequency of feed medium $(F M)$ every day $(1 / 1)$, every $2^{\mathrm{d}}(1 / 2)$ or $3^{\mathrm{d}}$ day $(1 / 3)$ of the same total feed medium volume and the effect of diluting 1.5 (concentrated FM) versus 2.5 times over the whole fed-batch cultivation while adding the same amount of nutrients. It was observed that the combination of PEP G and E gave higher productivity than the combination of PEP D and G in the base medium, 
feeding every $3^{\mathrm{d}}$ days was better than feeding every $2^{\mathrm{d}}$ day for the cell growth and the productivity, feeding every day seemed to help maintaining the viability but the productivity was similar as feeding every $2^{\mathrm{d}}$ day and finally higher cultivation dilution 2.5 versus 1.5 was more favourable to the viability and the productivity, see Figures 1 to 3.
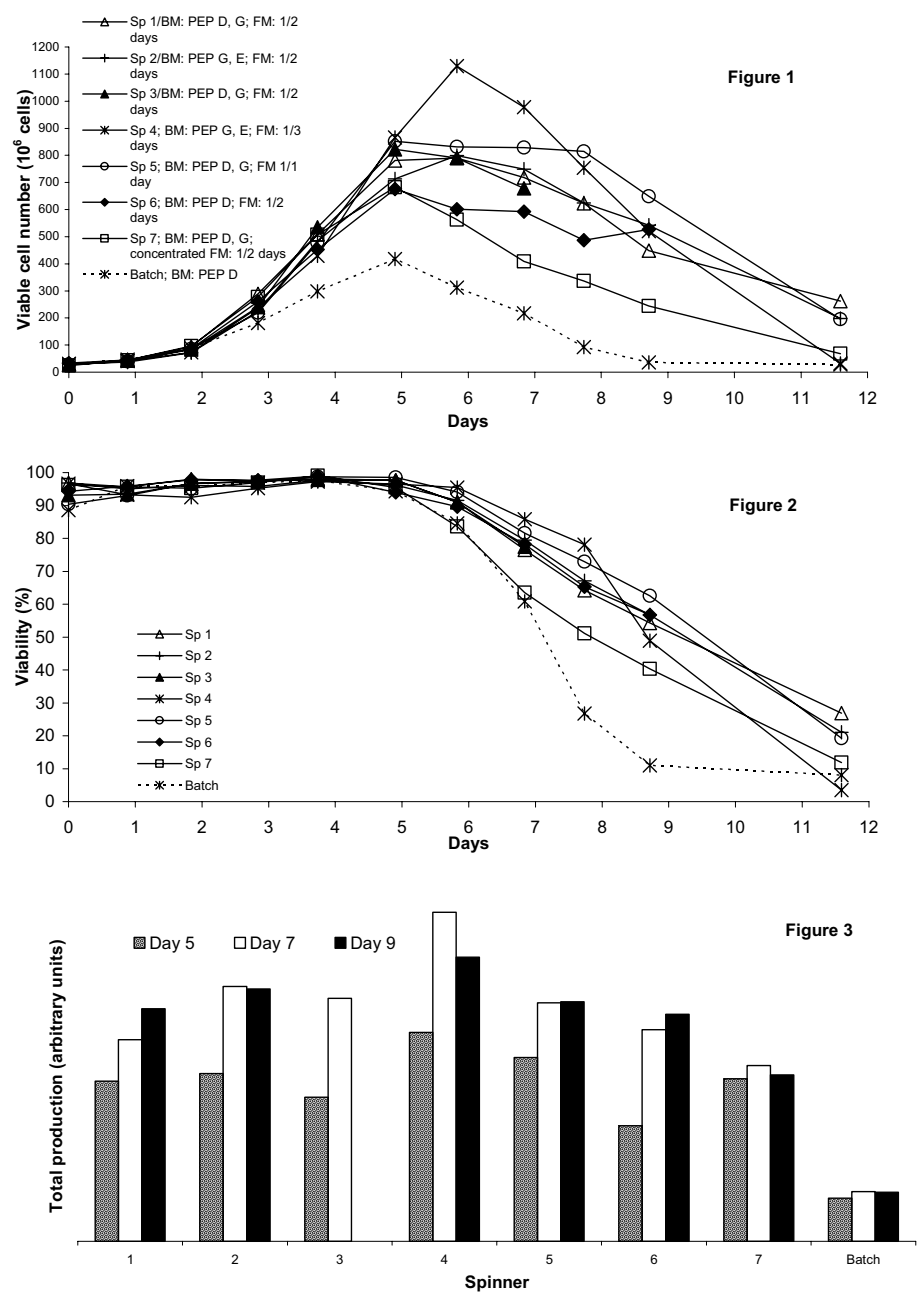

Figure 1-3. Viable cell number, viability and product accumulation in fed-batch spinners. 


\section{CONCLUSIONS}

A series of 8 plant peptones and 1 yeast peptones was studied together with a meat- and milk-derived peptones as references. 3 plant peptones were selected for further fed-batch process investigation due to their positive effect on the cell density, viability or productivity. The fed-batch strategy was improved by the use of plant peptones for this cell line. It was found that the amino acids should not be fed simultaneously with peptones $\mathrm{D}$ and $\mathrm{E}$ and the feed medium. This peptone combination was detrimental to the fed-batch process even if the individual peptones were beneficial to the process and the same combination had not been detrimental in batch screening. Different peptones had different effect on the cell growth, the viability and the productivity so that combining peptones was beneficial to the process; the best peptone combination was identified. A larger dilution of the cultivation brought by the feeding was beneficial.

\section{ACKNOWLEDGEMENTS}

We thank the company Symphogen, Denmark, for their kind permission to use their cell line. We also want to thank Dr. Sören Bregenholt and Dr. Anne Bondgaard Tolstrup for their kind support to get this permission and helping us in the publication release of this work. Our thanks as well to J. Mühr, K. Engström, J. Kinnander and U. Oswaldsson for the analytical support at Biovitrum. Finally, we address our thanks to the company Kerry, which provided graciously the Kerry/Quest peptones. 